\title{
Solving Systems of Polynomial Equations
}

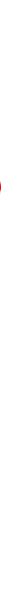




\section{Overview}

(1) Introduction

(2) A Simple Example

(3) Results

(4) Change of Basis

(5) An Example in $\mathbb{C}^{3}$

(6) Conclusions 


\section{Multivariate Polynomial Systems}

\section{Problem}

Find all vectors $\left(x_{1}, x_{2}, \ldots, x_{s}\right)^{\top} \in \mathbb{C}^{s}$ that satisfy

$$
\left\{\begin{array}{c}
p_{1}\left(x_{1}, x_{2}, \ldots, x_{s}\right)=0 \\
p_{2}\left(x_{1}, x_{2}, \ldots, x_{s}\right)=0 \\
\vdots \\
p_{s}\left(x_{1}, x_{2}, \ldots, x_{s}\right)=0
\end{array}\right.
$$

where $p_{i}\left(x_{1}, x_{2}, \ldots, x_{s}\right), 1 \leq i \leq s$ are polynomials.

Focus on the bivariate case: $s=2$

$$
\left\{\begin{array}{l}
p(x, y)=0 \\
q(x, y)=0
\end{array}\right.
$$




\section{Applications}

Multivariate polynomial systems find their applications in numerous fields of science and engineering:

- Robotics

- Computer graphics

- Polynomial optimization

- Signal processing

- Filter design

- Chemical engineering

- System identification

- Computer aided design

- ... 


\section{Existing Methods}

Contouring algorithms
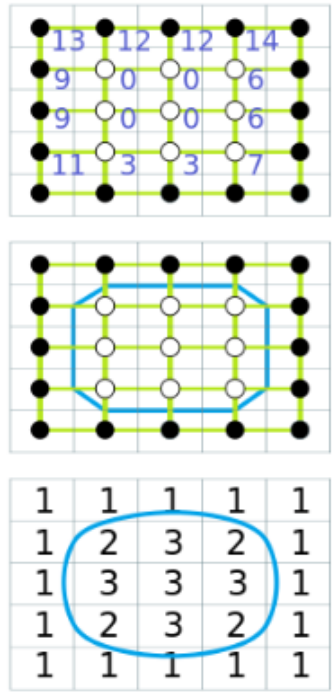

Wikipedia - Marching squares 


\section{Existing Methods}

Contouring algorithms

Groebner bases

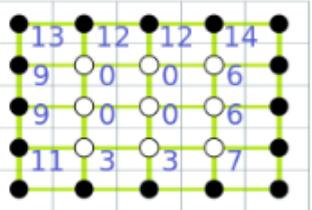

$$
\left\{\begin{array} { l } 
{ x y - 2 y = 0 } \\
{ 2 y ^ { 2 } - x ^ { 2 } = 0 }
\end{array} \rightarrow \left\{\begin{array}{l}
-2 x^{2}+x^{3}=0 \\
x y-2 y=0 \\
2 y^{2}-x^{2}=0
\end{array}\right.\right.
$$
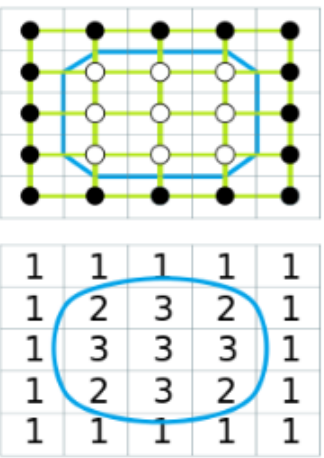

Wikipedia - Marching squares 


\section{Existing Methods}

Contouring algorithms

Groebner bases

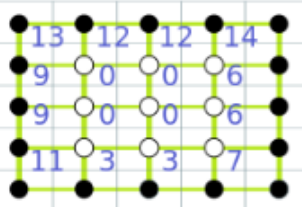

$$
\left\{\begin{array} { l } 
{ x y - 2 y = 0 } \\
{ 2 y ^ { 2 } - x ^ { 2 } = 0 }
\end{array} \rightarrow \left\{\begin{array}{l}
-2 x^{2}+x^{3}=0 \\
x y-2 y=0 \\
2 y^{2}-x^{2}=0
\end{array}\right.\right.
$$

Homotopy continuation
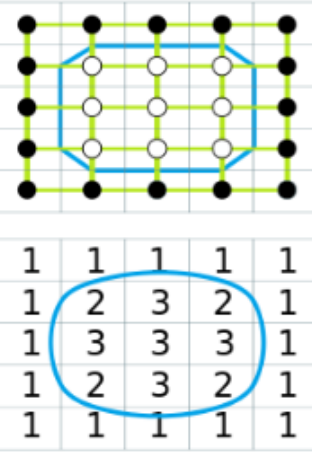

Wikipedia - Marching squares

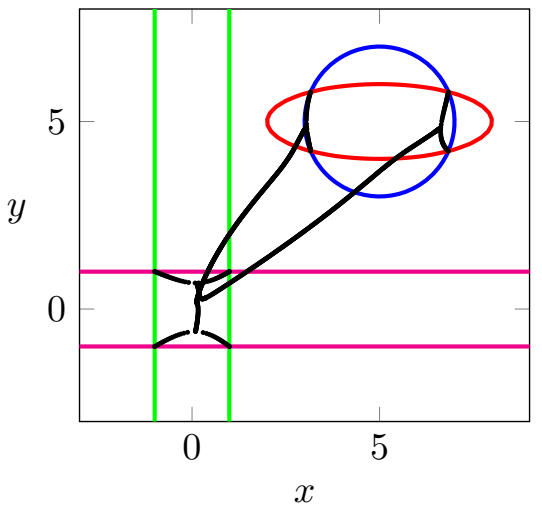




\section{Existing Methods}

Resultant methods
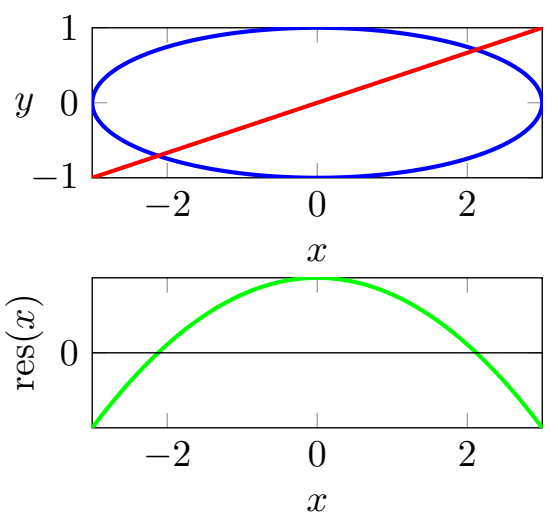


\section{Existing Methods}

2-Parameter eigenvalue problem

Resultant methods
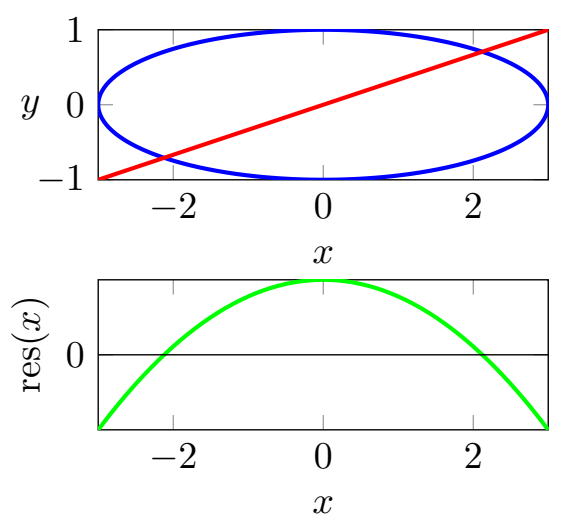

$$
\begin{gathered}
\operatorname{det}\left(A_{p}-x B_{p}-y C_{p}\right)=p(x, y) \\
\operatorname{det}\left(A_{q}-x B_{q}-y C_{q}\right)=q(x, y) \\
\left\{\begin{array}{c}
\left(A_{p}-x B_{p}-y C_{p}\right) \boldsymbol{u}_{p}=\mathbf{0} \\
\left(A_{q}-x B_{q}-y C_{q}\right) \boldsymbol{u}_{q}=\mathbf{0}
\end{array}\right. \\
\Delta_{0}=B_{p} \otimes C_{q}-C_{p} \otimes B_{q}, \\
\Delta_{1}=C_{p} \otimes A_{q}-A_{p} \otimes C_{q}, \\
\Delta_{2}=A_{p} \otimes B_{q}-B_{p} \otimes A_{q}, \\
\Delta_{1} \boldsymbol{w}=x \Delta_{0} \boldsymbol{w}, \\
\Delta_{2} \boldsymbol{w}=y \Delta_{0} \boldsymbol{w} . \\
\Rightarrow \mathcal{O}\left(\delta^{12}\right)
\end{gathered}
$$




\section{A Simple Example}

$$
\left\{\begin{array}{l}
p(x, y)=1-x y=0 \\
q(x, y)=2 x-x^{2}-2 y+y^{2}=0
\end{array}\right.
$$

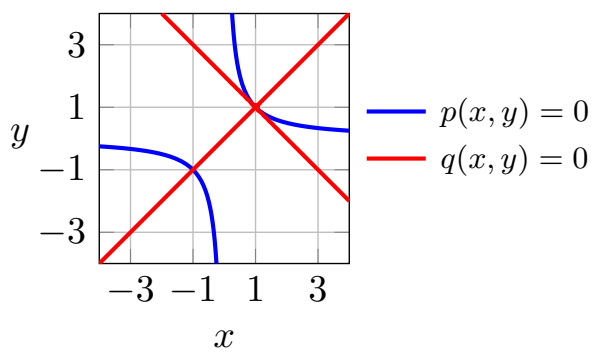




\section{A Simple Example}

$\left\{\begin{array}{l}p(x, y)=1-x y=0 \\ q(x, y)=2 x-x^{2}-2 y+y^{2}=0\end{array}\right.$
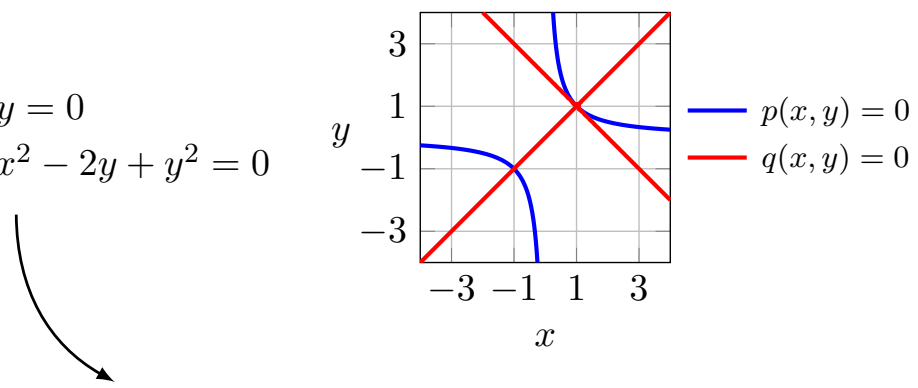

$$
\left(\begin{array}{ccc|cc|c}
1 & 0 & 0 & 0 & -1 & 0 \\
0 & 2 & -1 & -2 & 0 & 1
\end{array}\right)\left(\begin{array}{c}
1 \\
x \\
x^{2} \\
y \\
x y \\
y^{2}
\end{array}\right)=\left(\begin{array}{l}
p(x, y) \\
q(x, y)
\end{array}\right)=\left(\begin{array}{l}
0 \\
0
\end{array}\right)
$$




\section{A Simple Example}

$$
\begin{aligned}
& \left(\begin{array}{ccc|cc|c}
1 & 0 & 0 & 0 & -1 & 0 \\
0 & 2 & -1 & -2 & 0 & 1
\end{array}\right)\left(\begin{array}{c}
1 \\
x \\
\frac{x^{2}}{y} \\
\frac{x y}{y^{2}}
\end{array}\right)=\left(\begin{array}{l}
p(x, y) \\
q(x, y)
\end{array}\right)=\left(\begin{array}{l}
0 \\
0
\end{array}\right) \\
& \left(\begin{array}{ccc|cc|c}
1 & 0 & 0 & 0 & -1 & 0 \\
0 & 2 & -1 & -2 & 0 & 1 \\
\hline-x & 1 & & & & \\
& -x & 1 & & & \\
& & & -x & 1 & \\
\hline-y & & & 1 & &
\end{array}\right)\left(\begin{array}{c}
1 \\
x \\
x^{2} \\
y \\
\frac{x y}{y^{2}}
\end{array}\right)=\left(\begin{array}{c}
p(x, y) \\
\frac{q(x, y)}{0} \\
0 \\
0 \\
\hline 0 \\
0
\end{array}\right)=\mathbf{0} \\
& \rightarrow L(x, y) \boldsymbol{v}=\mathbf{0}
\end{aligned}
$$




\section{A Two-Parameter Eigenvalue Problem}

$$
\begin{aligned}
& \left(\begin{array}{ccc|cc|c}
1 & 0 & 0 & 0 & -1 & 0 \\
\hline-x & 1 & & & & \\
& -x & 1 & & & \\
\hline-y & & & -x & 1 & \\
\hline & & -y & & 1
\end{array}\right)\left(\begin{array}{c}
1 \\
x \\
\frac{x^{2}}{y} \\
\frac{x y}{y^{2}}
\end{array}\right)=\left(\begin{array}{ccc|cc|c}
0 & 2 & -1 & -2 & 0 & 1 \\
\hline-x & 1 & & & \\
& -x & 1 & & \\
& & & -x & 1 & \\
\hline-y & & & 1 & \\
& & & -y & 1
\end{array}\right)\left(\begin{array}{c}
1 \\
x \\
\frac{x^{2}}{y} \\
\frac{x y}{y^{2}}
\end{array}\right)=\mathbf{0} \\
& \left\{\begin{array}{l}
\left(A_{p}-x B_{p}-y C_{p}\right) \boldsymbol{u}_{p}=\mathbf{0} \\
\left(A_{q}-x B_{q}-y C_{q}\right) \boldsymbol{u}_{q}=\mathbf{0}
\end{array}\right.
\end{aligned}
$$




\section{Degree Extension}

\section{Property}

$$
\left\{\begin{array} { l } 
{ p ( x , y ) = 0 } \\
{ q ( x , y ) = 0 }
\end{array} \Leftrightarrow \left\{\begin{array}{l}
p(x, y)=0 \\
q(x, y)=0 \\
y p(x, y)=0
\end{array}\right.\right.
$$




\section{Degree Extension}

\section{Property}

$$
\left\{\begin{array} { l } 
{ p ( x , y ) = 0 } \\
{ q ( x , y ) = 0 }
\end{array} \Leftrightarrow \left\{\begin{array}{l}
p(x, y)=0 \\
q(x, y)=0 \\
y p(x, y)=0
\end{array}\right.\right.
$$

$$
\begin{aligned}
& p \rightarrow\left(\begin{array}{cccc|ccc|cc|c}
1 & 0 & 0 & 0 & 0 & -1 & 0 & 0 & 0 & 0 \\
0 & 2 & -1 & 0 & -2 & 0 & 0 & 1 & 0 & 0
\end{array}\right) \\
& \begin{array}{c}
q p \rightarrow \\
y p
\end{array} \rightarrow\left(\begin{array}{cccc|ccc|cc|c}
0 & 2 & -1 & 0 & -2 & 0 & 0 & 1 & 0 & 0 \\
1 & 0 & 0 & 0 & -1 & 0 \\
\hline-x & 1 & & & & & & & &
\end{array}\right)\left(\begin{array}{c}
1 \\
x \\
x^{2}
\end{array}\right) \\
& -x \quad 1 \\
& -x 1 \\
& \left.\begin{array}{l|ccc|cc|c}
-x & 1 & & & \\
& & -x & 1 & & \\
\hline-y & 1 & & -x & 1 & \\
-y & & 1 & \\
\frac{x^{2} y}{y^{2}} \\
x y^{2} \\
\hline y^{3}
\end{array}\right)
\end{aligned}
$$




\section{Pencil Reduction}

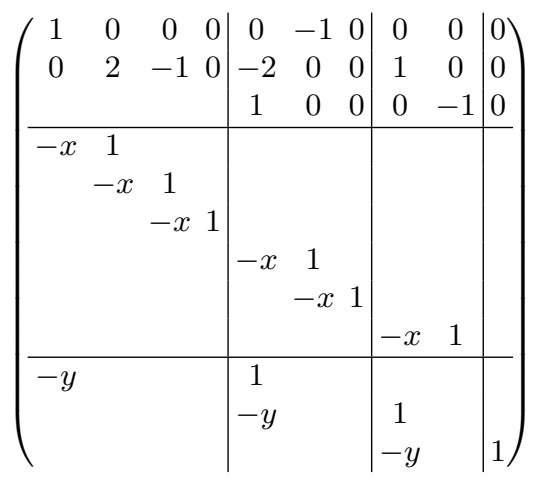




\section{Pencil Reduction}

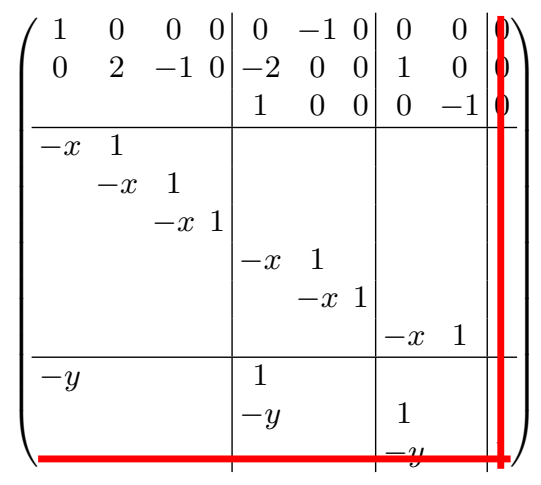




\section{Pencil Reduction}
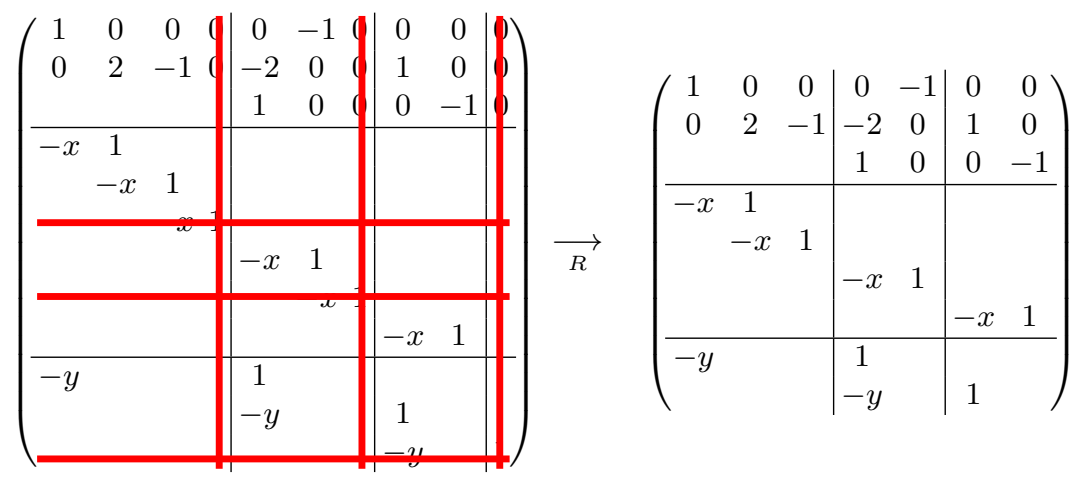

$$
\rightarrow \hat{L}_{r}(x, y) \boldsymbol{v}=\mathbf{0}
$$




\section{Pencil Reduction}

$$
\hat{\Pi}_{x, r}(x) \boldsymbol{v}=\mathbf{0}
$$

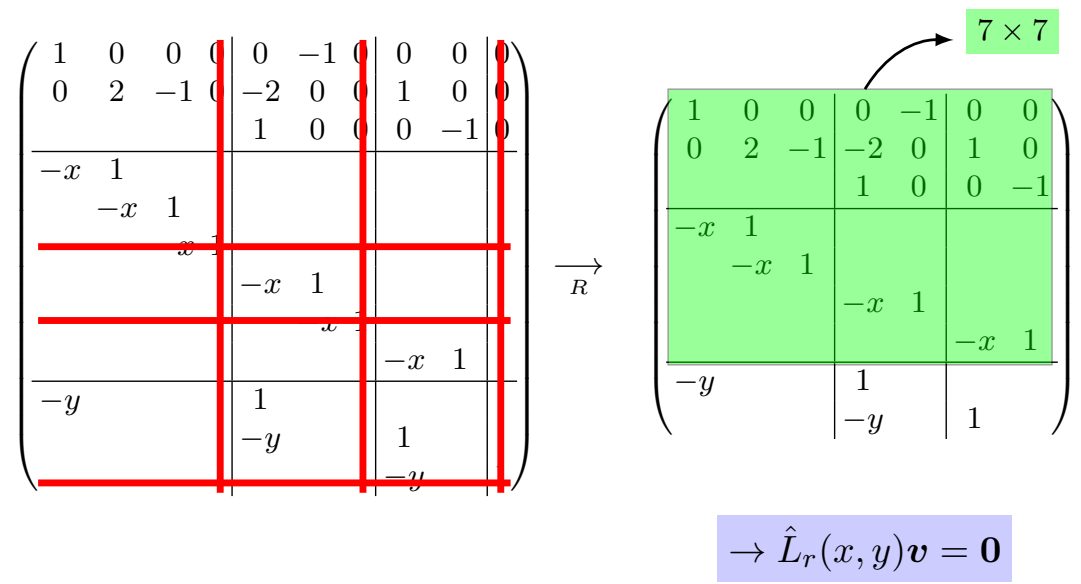




\section{Theorem}

In the degree extension step, add all equations $\left\{y^{i} p(x, y)=0\right\}_{1 \leq i<\delta_{q}^{y}}$ and $\left\{y^{i} q(x, y)=0\right\}_{1 \leq i<\delta_{p}^{y}}$ to the original system $p(x, y)=q(x, y)=0$. The resulting $x$-pencil $\hat{\Pi}_{x, r}(x)$ is square and

$$
\operatorname{det} \hat{\Pi}_{x, r}(x)=\gamma \operatorname{det} S^{p, q}(x)
$$

with $\gamma \in\{-1,1\}$.

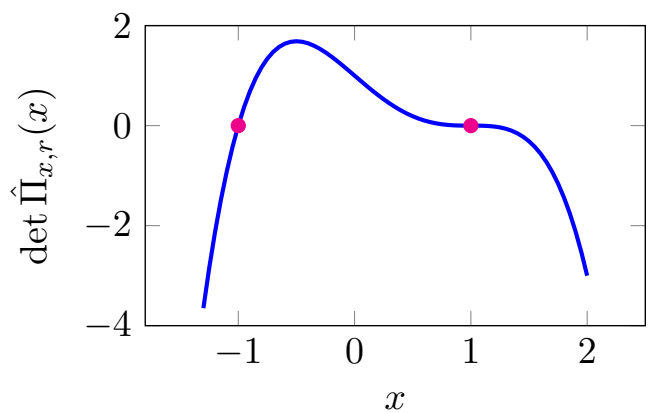

$\rightarrow x$-coordinates are obtained with correct multiplicities! 


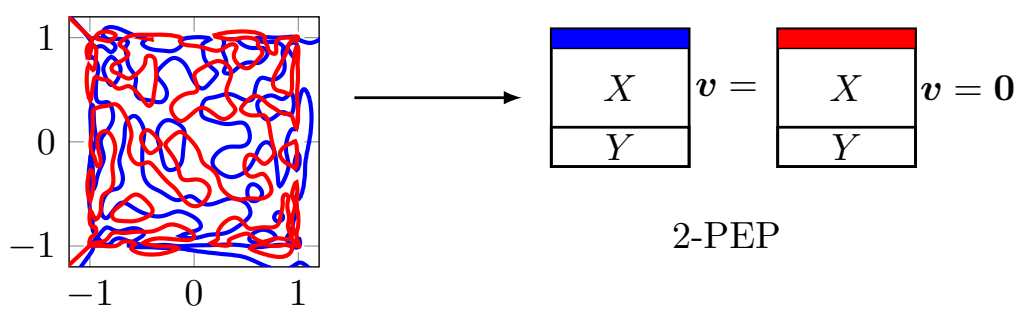



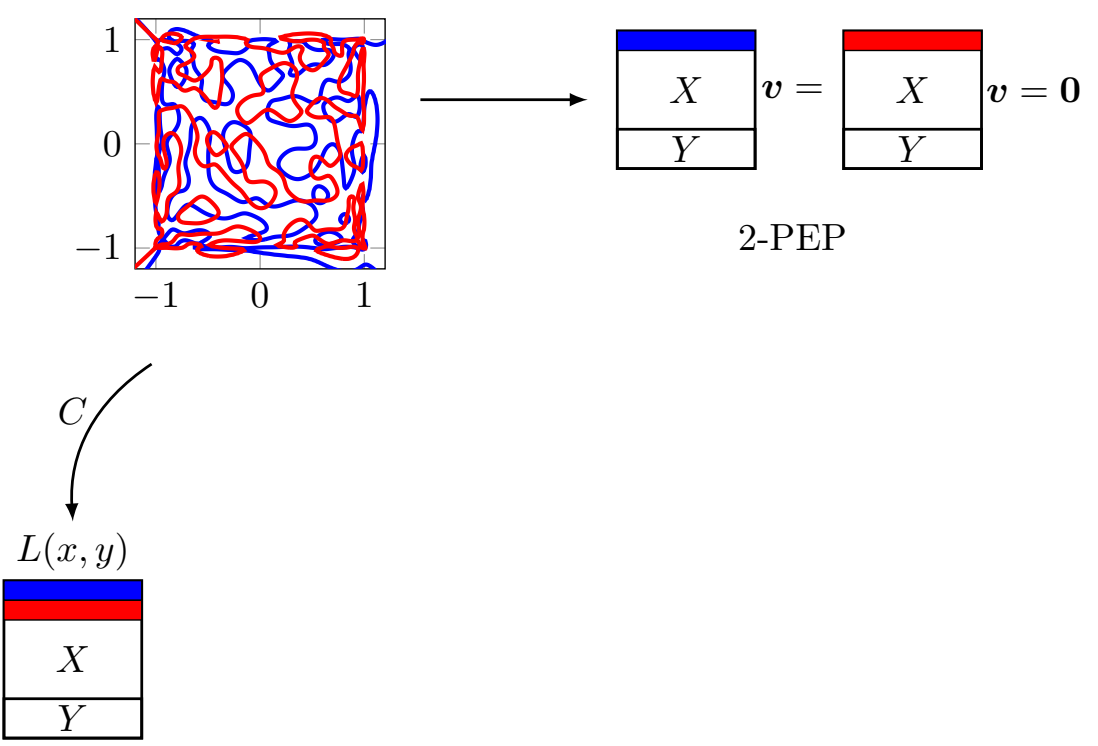

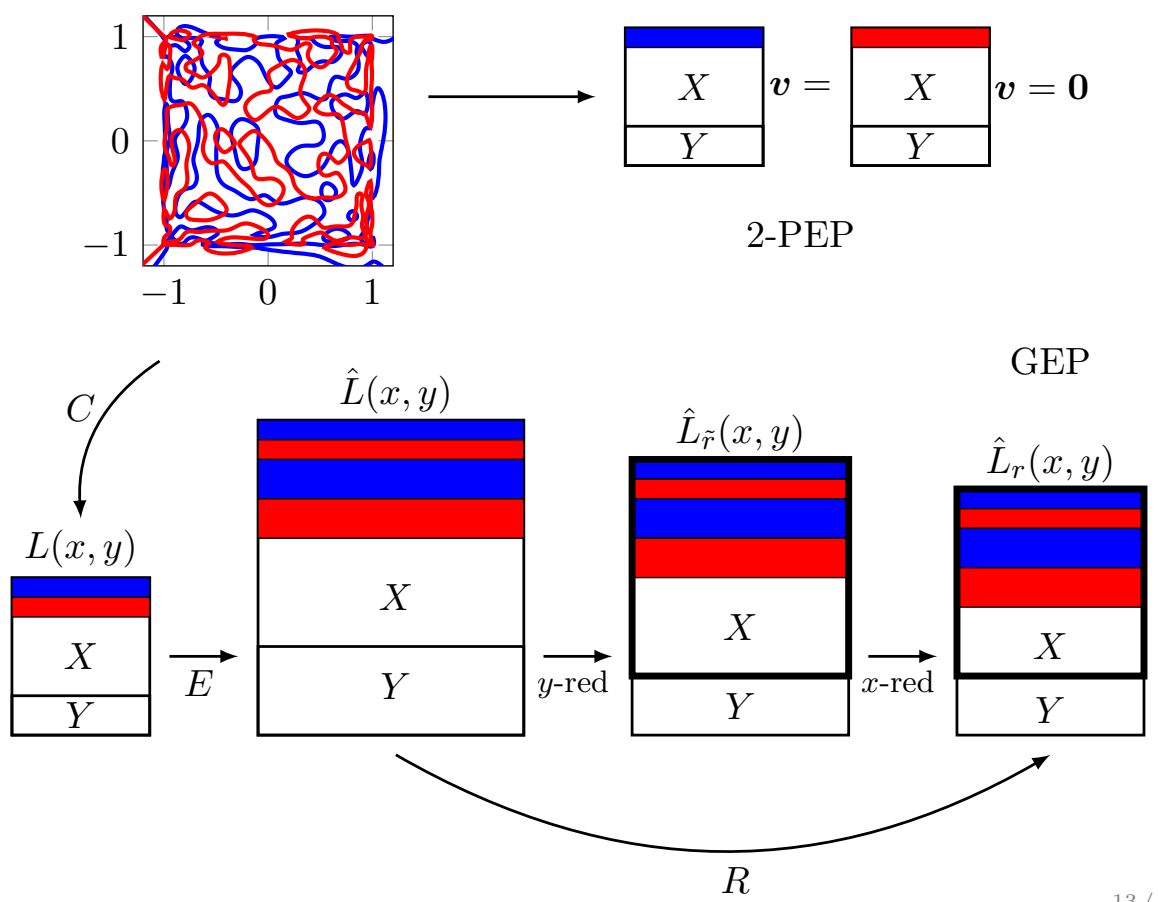


\section{Finding the $y$-coordinates}

Without solving an analogous GEP in $y$

- Eigenvectors of $\hat{\Pi}_{x, r}(x)$

- Common roots of $p\left(x^{*}, y\right)$ and $q\left(x^{*}, y\right)$

- Eigenvalues of $L\left(x^{*}, y\right)$

- A variable precision approach 


\section{Finding the $y$-coordinates}

Without solving an analogous GEP in $y$

- Eigenvectors of $\hat{\Pi}_{x, r}(x)$

- Common roots of $p\left(x^{*}, y\right)$ and $q\left(x^{*}, y\right)$

- Eigenvalues of $L\left(x^{*}, y\right)$

- A variable precision approach

Solving an analogous GEP in $y$ : a coupling of $x$ - and $y$-values based on the residual matrix
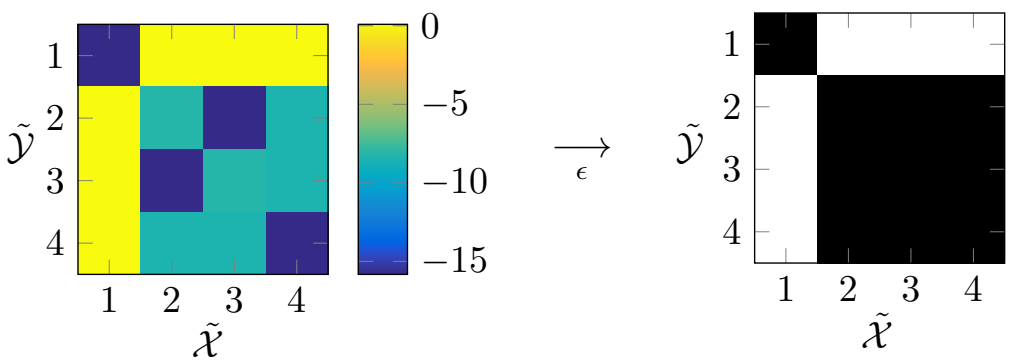


\section{Finding the $y$-coordinates}

- The eigenvectors of $\hat{\Pi}_{x, r}(x)$

$$
\begin{aligned}
\operatorname{null}\left(\hat{\Pi}_{x, r}(-1)\right)=\operatorname{span}\left\{\left(\begin{array}{lllllll}
1 & -1 & 1 & -1 & 1 & 1 & -1
\end{array}\right)^{\top}\right\} \\
\left.\left(\begin{array}{lllllll}
1 & x & x^{2} & y & x y & y^{2} & x y^{2}
\end{array}\right)\right|_{(-1,-1)}
\end{aligned}
$$

$\operatorname{null}\left(\hat{\Pi}_{x, r}(1)\right)=\operatorname{span}\left\{\left(\begin{array}{lllllll}1 & 1 & 1 & 1 & 1 & 1 & 1\end{array}\right)^{\top}\right\}$

$$
\left(\begin{array}{lllllll}
1 & x & x^{2} & y & x y & y^{2} & x y^{2}
\end{array}\right)
$$




\section{Finding the $y$-coordinates}

- Common roots of $p\left(x^{*}, y\right)$ and $q\left(x^{*}, y\right)$

$$
\begin{gathered}
p(-1, y)=1+y \\
q(-1, y)=-3-2 y+y^{2} \\
\left(\begin{array}{ccc}
1 & 1 & \\
& 1 & 1 \\
-3 & -2 & 1
\end{array}\right)\left(\begin{array}{c}
1 \\
y \\
y^{2}
\end{array}\right)=\mathbf{0} \\
\downarrow \\
y=-1
\end{gathered}
$$$$
p(1, y)=1-y
$$$$
q(1, y)=1-2 y+y^{2}
$$$$
\left(\begin{array}{ccc}
1 & -1 & \\
& 1 & -1 \\
1 & -2 & 1
\end{array}\right)\left(\begin{array}{c}
1 \\
y \\
y^{2}
\end{array}\right)=\mathbf{0}
$$

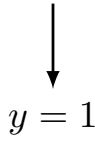




\section{Finding the $y$-coordinates}

- Eigenvalues of $L\left(x^{*}, y\right)$ : the values of $y$ for which $L\left(x^{*}, y\right)$ loses full column rank

$$
\begin{aligned}
& L(-1, y)=\left(\begin{array}{ccc|cc|c}
1 & 0 & 0 & 0 & -1 & 0 \\
0 & 2 & -1 & -2 & 0 & 1 \\
\hline 1 & 1 & & & & \\
& 1 & 1 & & & \\
& & & 1 & 1 & \\
\hline-y & & & 1 & & \\
& & & -y & & 1
\end{array}\right) \\
& \longrightarrow y=-1
\end{aligned}
$$




\section{Coupling all $x$ - and $y$-values}

Toy solution set:

$$
S=\left\{\left(x_{1}, y_{1}\right),\left(x_{1}, y_{2}\right),\left(x_{2}, y_{2}\right),\left(x_{2}, y_{3}\right),\left(x_{3}, y_{1}\right),\left(x_{3}, y_{4}\right)\right\}
$$

with $\mu_{11}=3, \mu_{12}=\mu_{23}=2, \mu_{22}=\mu_{31}=\mu_{34}=1$. 


\section{Coupling all $x$ - and $y$-values}

Toy solution set:

$$
S=\left\{\left(x_{1}, y_{1}\right),\left(x_{1}, y_{2}\right),\left(x_{2}, y_{2}\right),\left(x_{2}, y_{3}\right),\left(x_{3}, y_{1}\right),\left(x_{3}, y_{4}\right)\right\}
$$

with $\mu_{11}=3, \mu_{12}=\mu_{23}=2, \mu_{22}=\mu_{31}=\mu_{34}=1$.

Residual matrix $R(\mathcal{X}, \mathcal{Y})$

$x_{1}$
$x_{1}$
$y_{1}$
$y_{1}$
$y_{1}$
$y_{2}$
$y_{2}$
$y_{2}$
$y_{3}$
$y_{3}$
$y_{4}$$\left(\begin{array}{ccccccccccc}0 & 0 & 0 & 0 & 0 & \times & \times & \times & 0 & 0 \\ 0 & 0 & 0 & 0 & 0 & \times & \times & \times & 0 & 0 \\ 0 & 0 & 0 & 0 & 0 & \times & \times & \times & 0 & 0 \\ 0 & 0 & 0 & 0 & 0 & \times & \times & \times & 0 & 0 \\ 0 & 0 & 0 & 0 & 0 & 0 & 0 & 0 & \times & \times \\ 0 & 0 & 0 & 0 & 0 & 0 & 0 & 0 & \times & \times \\ 0 & 0 & 0 & 0 & 0 & 0 & 0 & 0 & \times & \times \\ \times & \times & \times & \times & \times & 0 & 0 & 0 & \times & \times \\ \times & \times & \times & \times & \times & 0 & 0 & 0 & \times & \times \\ \times & \times & \times & \times & \times & \times & \times & \times & 0 & 0\end{array}\right)$




\section{Coupling all $x$ - and $y$-values}

Toy solution set:

$$
S=\left\{\left(x_{1}, y_{1}\right),\left(x_{1}, y_{2}\right),\left(x_{2}, y_{2}\right),\left(x_{2}, y_{3}\right),\left(x_{3}, y_{1}\right),\left(x_{3}, y_{4}\right)\right\}
$$

with $\mu_{11}=3, \mu_{12}=\mu_{23}=2, \mu_{22}=\mu_{31}=\mu_{34}=1$.

Residual matrix $R(\mathcal{X}, \mathcal{Y})$

$x_{1}$
$y_{1}$
$y_{1}$
$y_{1}$
$y_{1}$
$y_{2}$
$y_{2}$
$y_{2}$
$y_{3}$
$y_{3}$
$y_{4}$$\left(\begin{array}{cccccccccc}0 & 0 & 0 & 0 & 0 & \times & \times & \times & 0 & 0 \\ 0 & 0 & 0 & 0 & 0 & \times & \times & \times & 0 & 0 \\ 0 & 0 & 0 & 0 & 0 & \times & \times & \times & 0 & 0 \\ 0 & 0 & 0 & 0 & 0 & \times & \times & \times & 0 & 0 \\ 0 & 0 & 0 & 0 & 0 & 0 & 0 & 0 & \times & \times \\ 0 & 0 & 0 & 0 & 0 & 0 & 0 & 0 & \times & \times \\ 0 & 0 & 0 & 0 & 0 & 0 & 0 & 0 & \times & \times \\ \times & \times & \times & \times & \times & 0 & 0 & 0 & \times & \times \\ \times & \times & \times & \times & \times & 0 & 0 & 0 & \times & \times \\ \times & \times & \times & \times & \times & \times & \times & \times & 0 & 0\end{array}\right)$

$\left(c_{1}^{x}\right)$

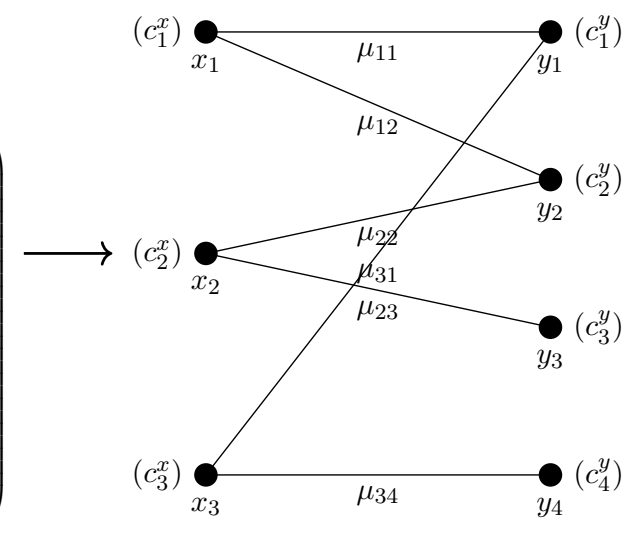




\section{Coupling all $x$ - and $y$-values}

Finding the multiplicities from the feasibility equations:

$$
\begin{aligned}
& \left\{\begin{array}{l}
\mu_{11}+\mu_{12}=c_{1}^{x} \\
\mu_{22}+\mu_{23}=c_{2}^{x} \\
\mu_{31}+\mu_{34}=c_{3}^{x} \\
\mu_{11}+\mu_{31}=c_{1}^{y} \\
\mu_{12}+\mu_{22}=c_{2}^{y} \\
\mu_{23}=c_{3}^{y} \\
\mu_{34}=c_{4}^{y}
\end{array} \rightarrow\left(\begin{array}{llllll}
1 & 1 & & & & \\
& & 1 & 1 & & \\
& & & & 1 & 1 \\
1 & & & & 1 & \\
& 1 & 1 & & & \\
& & & 1 & & \\
& & & & &
\end{array}\right)\left(\begin{array}{l}
\mu_{11} \\
\mu_{12} \\
\mu_{22} \\
\mu_{23} \\
\mu_{31} \\
\mu_{34}
\end{array}\right)=\left(\begin{array}{l}
c_{1}^{x} \\
c_{2}^{x} \\
c_{3}^{x} \\
c_{1}^{y} \\
c_{2}^{y} \\
c_{3}^{y} \\
c_{4}^{y}
\end{array}\right) \rightarrow M \boldsymbol{\mu}=\boldsymbol{c}\right. \\
& \longrightarrow \mu_{11}=3, \mu_{12}=\mu_{23}=2, \mu_{22}=\mu_{31}=\mu_{34}=1 .
\end{aligned}
$$




\section{Coupling all $x$ - and $y$-values}

Finite precision:

$$
\begin{aligned}
& \tilde{x}_{11} \tilde{x}_{12} \tilde{x}_{13} \tilde{x}_{14} \tilde{x}_{15} \tilde{x}_{21} \tilde{x}_{22} \tilde{x}_{23} \tilde{x}_{31} \tilde{x}_{32} \\
& \begin{array}{l}
\tilde{y}_{11} \\
\tilde{y}_{12}
\end{array}\left(\begin{array}{cccccccccc}
\tilde{0} & \tilde{0} & \tilde{0} & \tilde{0} & \tilde{0} & \times & \times & \times & \tilde{0} & \tilde{0} \\
\tilde{0} & \tilde{0} & \tilde{0} & \tilde{0} & \tilde{0} & \times & \times & \times & \tilde{0} & \tilde{0}
\end{array}\right. \\
& \begin{array}{lllllllllllllll}
\tilde{y}_{13} & \tilde{0} & \tilde{0} & \tilde{0} & \tilde{0} & \tilde{0} & \times & \times & \times & \tilde{0} & \tilde{0}
\end{array} \\
& \begin{array}{l|lllllllllll}
\tilde{y}_{14} & \tilde{0} & \tilde{0} & \tilde{0} & \tilde{0} & \tilde{0} & \times & \times & \times & \tilde{0} & \tilde{0}
\end{array} \\
& R(\tilde{\mathcal{X}}, \tilde{\mathcal{Y}})=\tilde{y}_{21} \quad \begin{array}{ccccccccccc}
0 & \tilde{0} & \tilde{0} & \tilde{0} & \tilde{0} & \tilde{0} & \tilde{0} & \tilde{0} & \times & \times
\end{array} \\
& \begin{array}{llllllllllll}
\tilde{y}_{22} & \tilde{0} & \tilde{0} & \tilde{0} & \tilde{0} & \tilde{0} & \tilde{0} & \tilde{0} & \tilde{0} & \times & \times
\end{array} \\
& \begin{array}{l|llllllllll}
\tilde{y}_{23} & \tilde{0} & \tilde{0} & \tilde{0} & \tilde{0} & \tilde{0} & \tilde{0} & \tilde{0} & \tilde{0} & \times & \times
\end{array} \\
& \begin{array}{lllllllllllllllllll}
\tilde{y}_{31} & \times & \times & \times & \times & \times & \tilde{0} & \tilde{0} & \tilde{0} & \times & \times
\end{array} \\
& \tilde{y}_{32} \times \quad \times \quad \times \quad \times \quad \times \quad \times \quad \begin{array}{ccccccccccc}
0 & \tilde{0} & \tilde{0} & \times & \times
\end{array} \\
& \left.\begin{array}{lllllllllllllll}
\tilde{y}_{41} & \times & \times & \times & \times & \times & \times & \times & \times & \tilde{0} & \tilde{0}
\end{array}\right)
\end{aligned}
$$




\section{Coupling all $x$ - and $y$-values}

Numerical connection diagram:

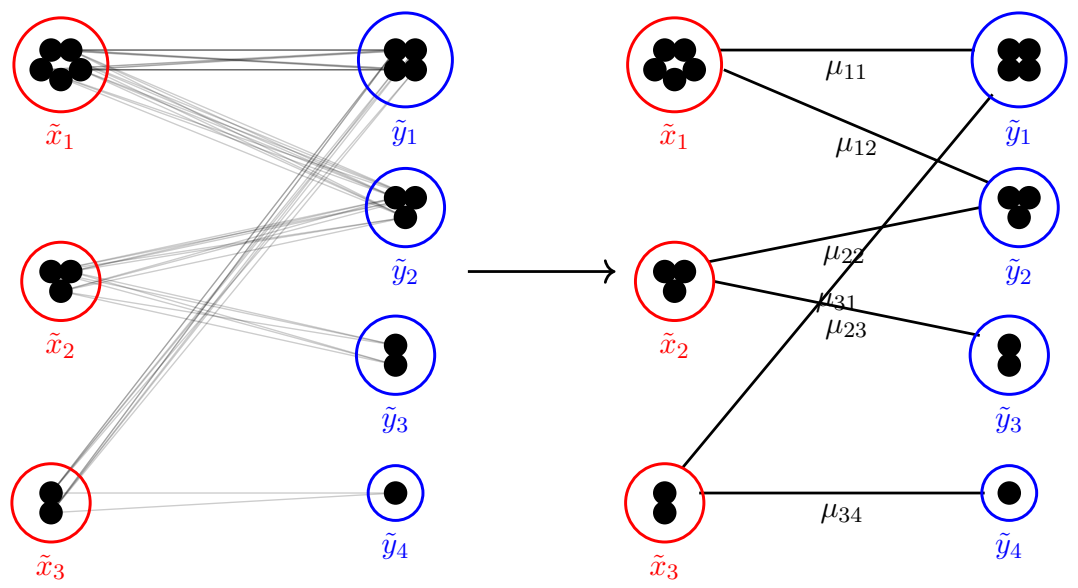

The clustered diagram leads to the same set of feasibility equations. 


\section{Results}
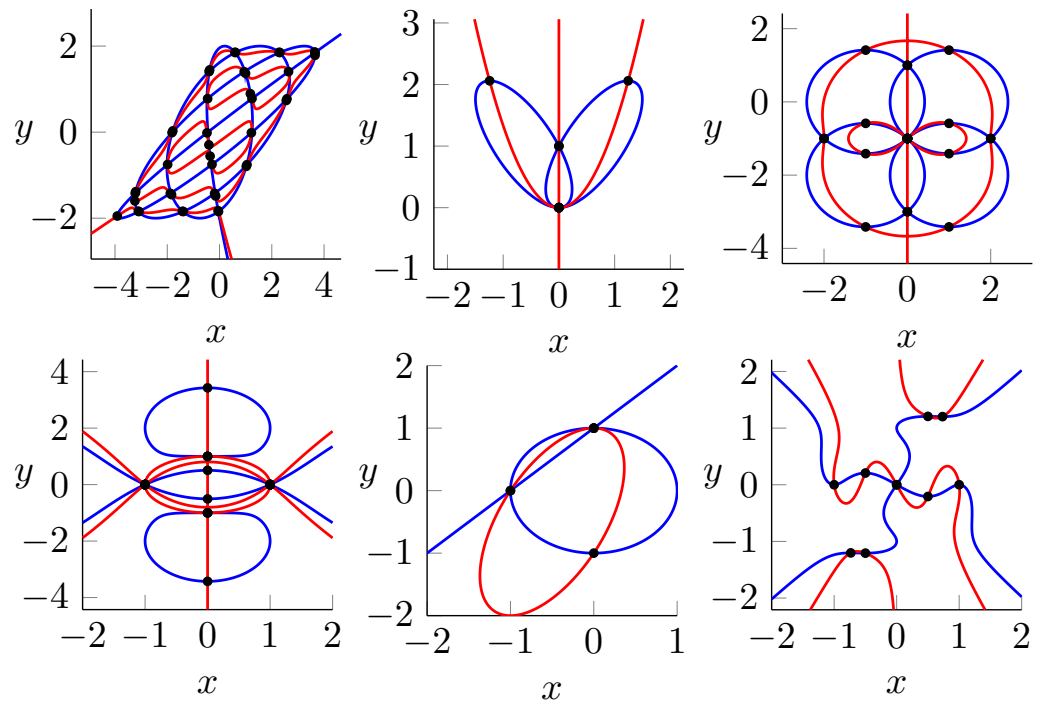


\section{Results}

Performance curve:

$$
\rho_{s}(\tau)=\frac{\left|\left\{p \in P \mid t_{p, s} \leq 2^{\tau}\left(\min _{s \in S} t_{p, s}\right)\right\}\right|}{|P|}
$$

PHClab (-), Bertini ( $(-)$, PNLA ( $(-)$ and our solver $(-)$.

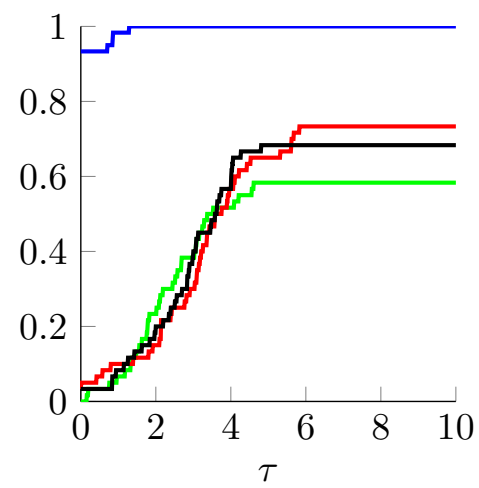




\section{Results}

Performance curve:

$$
\rho_{s}(\tau)=\frac{\left|\left\{p \in P \mid t_{p, s} \leq 2^{\tau}\left(\min _{s \in S} t_{p, s}\right)\right\}\right|}{|P|}
$$

PHClab (-), Bertini ( $(-)$, PNLA ( $(-)$ and our solver $(-)$.
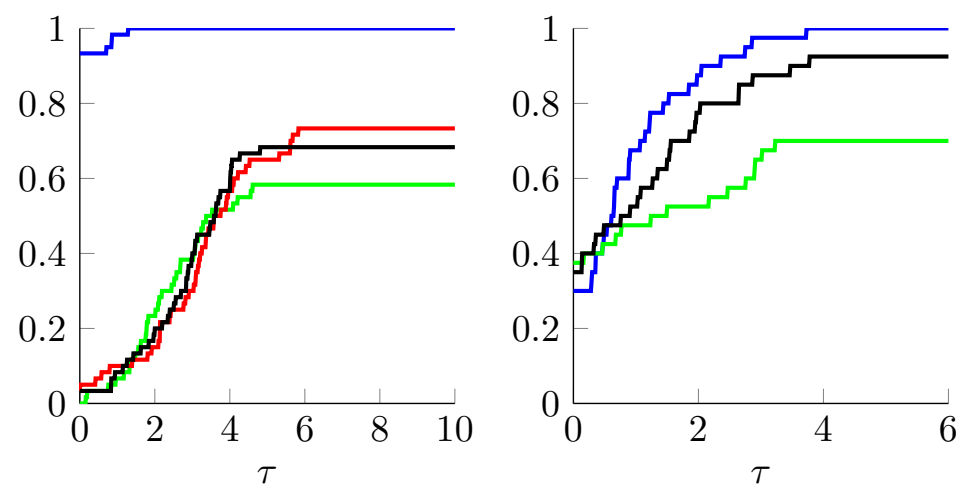


\section{Generic Problems}

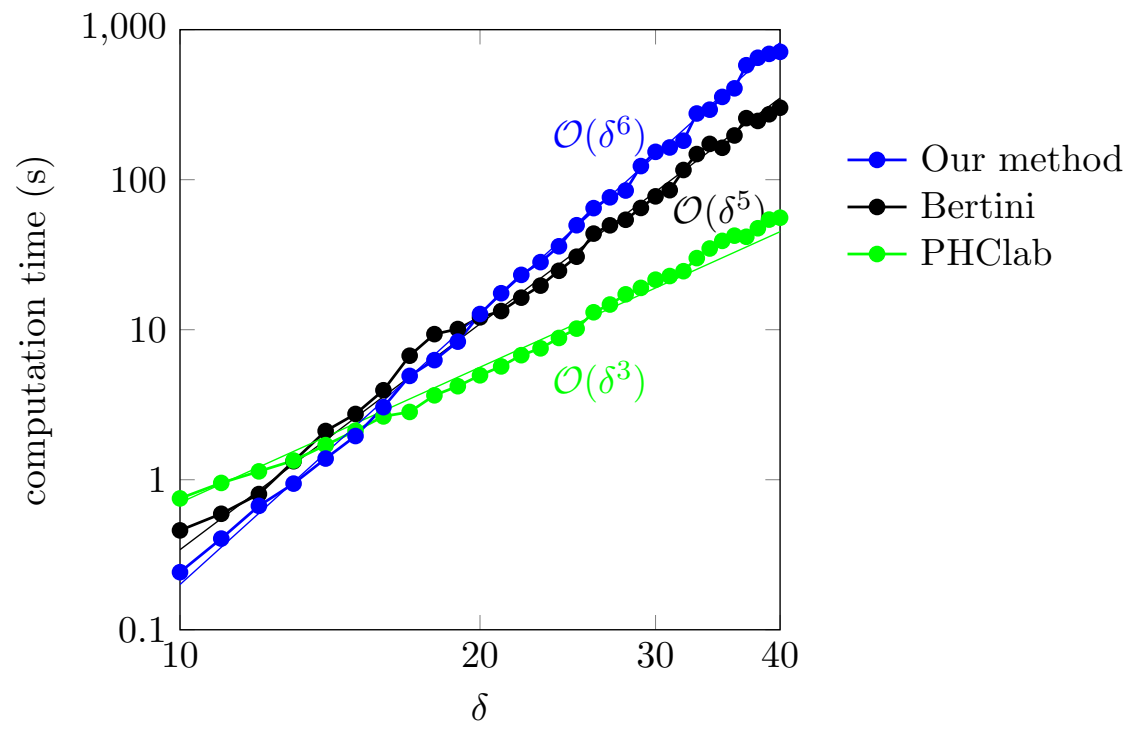




$$
\delta_{p}=\delta_{q}=40
$$

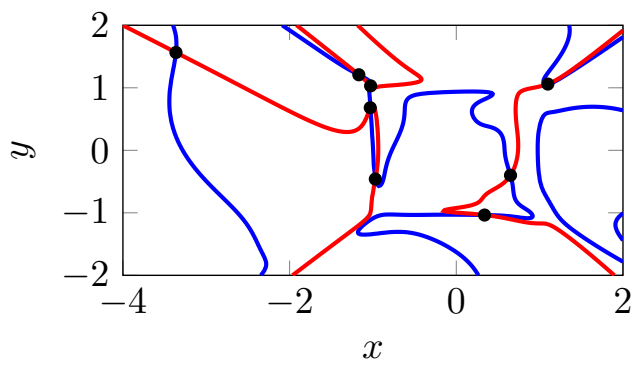

residuals before $(-)$ and after $(-)$ NR sweep

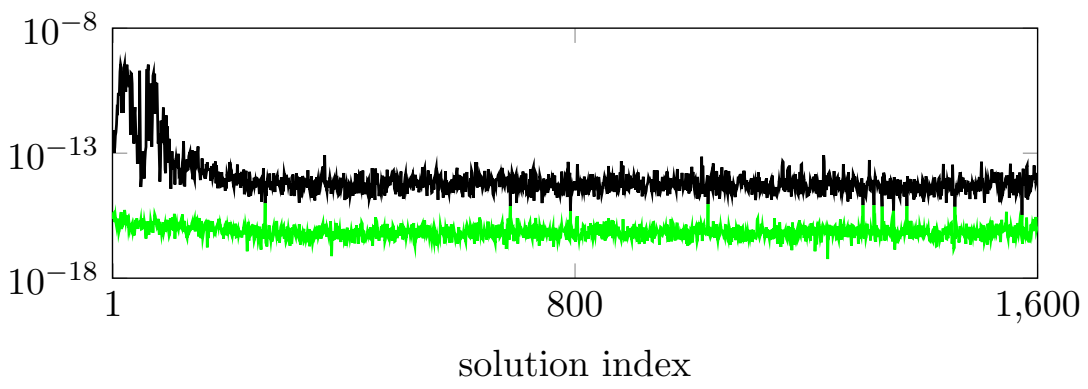




\section{A Change of Basis}

Legendre polynomials:

$$
\left\{\begin{array}{l}
P_{0}(x)=1 \\
P_{1}(x)=x \\
P_{n+1}(x)=\frac{2 n+1}{n+1} x P_{n}(x)-\frac{n}{n+1} P_{n-1}(x), \quad n \in \mathbb{N}_{0}
\end{array}\right.
$$

Example: $\left\{\begin{array}{l}p(x, y)=x^{2}+y^{2}-1=0 \\ q(x, y)=x y-1 / 4=0\end{array}\right.$

$\left(\begin{array}{ccc|cc|c}-\frac{1}{3} & 0 & \frac{2}{3} & 0 & 0 & \frac{2}{3} \\ -\frac{1}{4} & 0 & 0 & 0 & 1 & 0 \\ \hline-x & 1 & & & & \\ \frac{1}{2} & -\frac{3}{2} x & 1 & & & \\ & & & -x & 1 & \\ \hline-y & & & 1 & & \\ \frac{1}{2} & & & -\frac{3}{2} x & 1\end{array}\right)\left(\begin{array}{c}P_{0}(y) P_{0}(x) \\ P_{0}(y) P_{1}(x) \\ P_{0}(y) P_{2}(x) \\ P_{1}(y) P_{0}(x) \\ P_{1}(y) P_{1}(x) \\ \hline P_{2}(y) P_{0}(x)\end{array}\right)=\left(\begin{array}{c}p(x, y) \\ q(x, y) \\ 0 \\ 0 \\ 0 \\ 0 \\ 0\end{array}\right)$




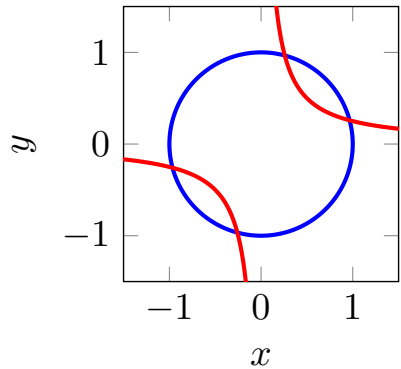

$$
\left\{\begin{array}{l}
x^{2}+y^{2}-1=0 \\
x y-1 / 4=0
\end{array}\right.
$$




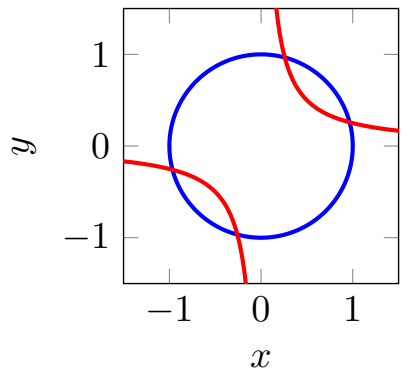

$$
\left\{\begin{array}{l}
x^{2}+y^{2}-1=0 \\
x y-1 / 4=0
\end{array}\right.
$$

$\operatorname{det} \hat{\Pi}_{x, r}(x)$

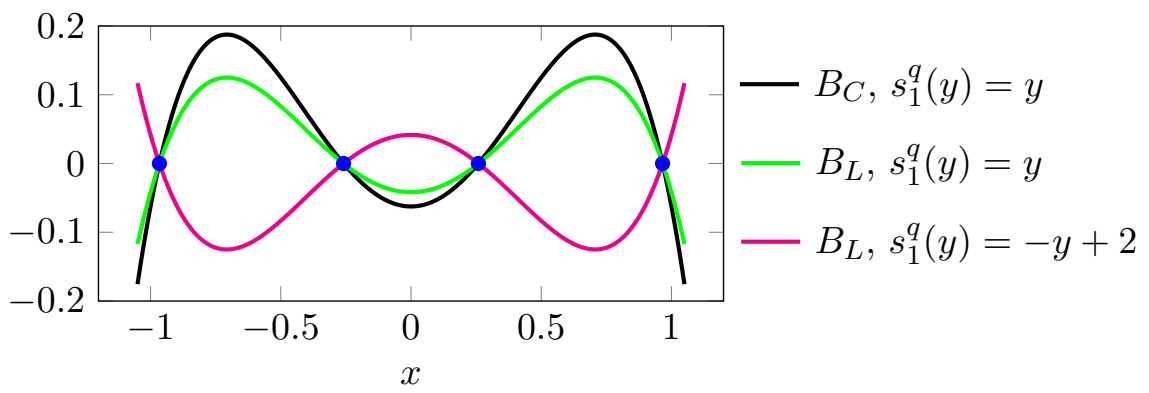




\section{An Example in $\mathbb{C}^{3}$}
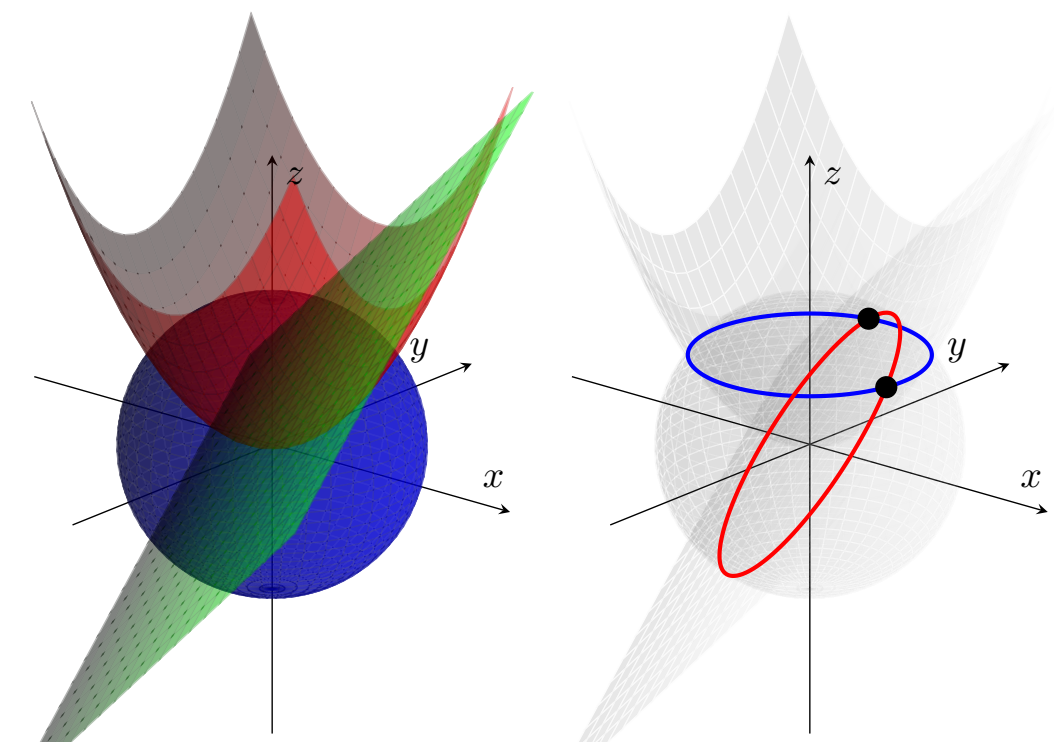


\section{Conclusions}

Conclusions

- From bivariate polynomial system to square generalized eigenvalue problem

- The pencil is constructed in an intuitive manner and shown to be equivalent to $S^{p, q}(x)$

- The polynomial coefficients appear directly in the pencil, without being manipulated 


\section{Conclusions}

Conclusions

- From bivariate polynomial system to square generalized eigenvalue problem

- The pencil is constructed in an intuitive manner and shown to be equivalent to $S^{p, q}(x)$

- The polynomial coefficients appear directly in the pencil, without being manipulated

- Moderate degree problems: accurate results and correct multiplicity information (in DP and without NR refinement)

- Generic problems: all solutions are found with small residuals, at least up to degree 40 


\section{Future work}

- Generalizations to higher dimensions

- The influence of the choice of basis on the accuracy

- Scaling

- Exploiting the (sparse) structure of $\hat{\Pi}_{x, r}(x)$ to speed up calculations

- Generic transformations of variables 


\section{References I}

K. Batselier. A Numerical Linear Algebra Framework for Solving Problems with Multivariate Polynomials. KU Leuven - Faculty of Engineering Science, 2013. PhD thesis, promotor: Bart De Moor.

L. Busé, H. Khalil, and B. Mourrain. Resultant-Based Methods for Plane Curves Intersection Problems. Springer-Verlag Berlin Heidelberg, 2016.

言 E. D. Dolan and J. J. Moré. Benchmarking optimization software with performance profiles. Mathematical Programming, 91:201-213, 2002.

言 P. Dreesen. Back to the Roots. KU Leuven - Faculty of Engineering Science, 2013. PhD thesis, promotor: Bart De Moor.

B. Buchberger. Groebner Bases: A short introduction for systems theorists. Computer Aided Systems Theory, 2178:1-19, 2002.

国

B. Plestenjak and M.E. Hochstenbach. Roots of bivariate polynomial systems via determinantal representations. SIAM J. Sci. Comput., 38(2):A765-A788, 2015. 


\section{References II}

A. J. Sommese and C. W. Wampler. The Numerical Solution of Systems of Polynomials Arising in Engineering Science. World Scientific Publishing Co. Pte. Ltd., 2005.

L. Sorber, M. Van Barel, and L. De Lathauwer. Numerical solution of bivariate and polyanalytic polynomial systems. SIAM J. Num. Anal. 52, pages 1551-1572, 2014.

击 J. Verschelde, Homotopy Continuation Methods for Solving Polynomial Systems. KU Leuven - Faculty of Engineering Science, 1996. PhD thesis, promotor: Ann Haegemans.

D. J. Bates, J. D. Hauenstein, A. J. Sommese, and C. W. Wampler. Numerically solving polynomial systems with Bertini, volume 25 of Software, Environments and Tools. SIAM, 2013. 